\title{
TRAUMATISMO ENCEFALOCRANEANO EN NIÑOS ATENDIDOS EN UN HOSPITAL NACIONAL DE LIMA, PERÚ 2004-2011
}

\author{
Daniel Guillén-Pinto ${ }^{1,2, a}$, Alonso Zea-Vera1,a, Daniel Guillén-Mendoza ${ }^{1, a}$, Miguel Situ-Kcomtt,a, \\ Carolina Reynoso-Osnayo ${ }^{1, b}$, Luis M. Milla-Vera ${ }^{1, c}$, Erika Bravo-Padilla ${ }^{3, d}$, Iván O. Espinoza ${ }^{1,2, a}$, \\ María del Pilar Medina-Alva1,4,a
}

\section{RESUMEN}

Con el objetivo de describir las características del traumatismo encéfalocraneano (TEC) en niños atendidos en el Hospital Nacional Cayetano Heredia entre los años 2004 y 2011 se realizó un estudio de serie de casos. Se incluyeron 316 menores de 14 años, la mediana de edad fue $4 \pm 3$ años. El 63,9\% fueron hombres. Las principales causas del TEC fueron las caídas $(80,1 \%)$, y los accidentes de tránsito $(10,4 \%)$. El 42,4\% (134/316) ocurrieron durante horas de la tarde. Hubo mayor frecuencia de accidentes en el hogar $(59,2 \%)$. El hematoma epidural $(26,9 \%)$ fue la lesión intracraneal más frecuente. Se requirió manejo en cuidados intensivos en $15,8 \%$ e intervenciones quirúrgicas en $14,2 \%$ de los casos. Se concluye que las caídas en el hogar fueron la causa principal de TEC y el hematoma epidural la lesión intracraneal más común donde son los menores de 5 años los más afectados.

Palabras clave: Traumatismos encefálicos; Niño; Accidentes domésticos; Accidentes por caídas; Hematoma epidural craneal (fuente: DeCS BIREME).

\section{TRAUMATIC BRAN INJURY IN CHILDREN ATTENDING A NATIONAL HOSPITAL IN LIMA, PERU 2004-2011}

\begin{abstract}
In order to describe the characteristics of traumatic brain injury (TEC) in children seen at the Cayetano Heredia National Hospital from 2004 to 2011, a case study was conducted. 316, 14 year-old minors were included, the median age was $4 \pm 3$ years old. $63.9 \%$ were male. The main causes of TEC were falls $(80.1 \%)$ and car accidents $(10.4 \%) .42 .4 \%(134 / 316)$ occurred in the afternoon. There was a higher incidence of falls at home (59.2\%). The epidural hematoma (26.9\%) was the most common intracraneal injury. Intensive care management and neurosurgical interventions were required in 15.8 and $14.2 \%$ of cases respectively. It is concluded that domestic falls were the main cause of accidents for TEC and epidural hematoma the most common intracranial injury where children under 5 were the most affected.
\end{abstract}

Key words: Brain injuries; Child; Accidents, home; Accidental falls; Hematoma, epidural, cranial (source: MeSH NLM).

\section{INTRODUCCIÓN}

El traumatismo encéfalocraneano (TEC) es causa importante de morbilidad y mortalidad (1-3). En Sudamérica se reporta la mayor incidencia y constituye una de las principales causas de muerte en menores de 15 años ${ }^{(4,5)}$. En Perú, el trauma es la primera causa de muerte en niños entre 1 y 14 años y el TEC corresponde al $57,3 \%$ de casos ${ }^{(6)}$. La mortalidad por TEC se produce en tres momentos, $50 \%$ fallecen inmediatamente después del accidente, $35 \%$ en la primera hora después del accidente y $15 \%$ durante la hospitalización. Por lo tanto, la prevención sería la forma más eficiente de disminuir la mortalidad ${ }^{(5)}$ y la mejor estrategia debiera ser a través de campañas de educación e investigación de los factores de riesgo para cada región (7).

Facultad de Medicina, Universidad Peruana Cayetano Heredia. Lima, Perú.

Unidad de Neuropediatría, Hospital Nacional Cayetano Heredia. Lima, Perú

Servicio de Neonatología, Hospital Nacional Cayetano Heredia. Lima, Perú.

4 Unidad de Neuropediatría, Instituto Nacional Materno Perinatal. Lima, Perú.

Neurólogo pediatra; ${ }^{\mathrm{b}}$ médico cirujano; ${ }^{\mathrm{c}}$ médico residente de neurología pediátrica; ${ }^{\mathrm{d}}$ médico pediatra.

* El resumen fue presentado en el XXVII Congreso Nacional de Pediatría, Arequipa, Perú. 2012.

Recibido: 26-04-2013 Aprobado: 02-10-13

Citar como: Guillén-Pinto D, Zea-Vera A, Guilén-Mendoza D, Situ-Kcomt M, Reynoso-Osnayo C, Miya-Vera LM, et al. Traumatismo encefalocraneano en niños atendidos en un hospital nacional de Lima, Perú 2004-2011. Rev Peru Med Exp Salud Publica. 2013;30(4):630-4. 
En la mayoría de países las caídas son la principal causa de TEC pediátrico (8-10); no obstante, los accidentes de tránsito tienen una mayor prevalencia en algunas regiones ${ }^{(5,11-13)}$. En la mayoría de países sudamericanos no se conocen las características propias del accidente, como días y meses más frecuentes, lugar, hora del día y mecanismo ${ }^{(7)}$. En nuestros hospitales, a pesar de ser una de las principales causas de atención en emergencia, no se conoce la evolución clínica de los niños hospitalizados y los estudios de referencia no pueden ser extrapolados a nuestra realidad. El objetivo del estudio fue describir las principales características epidemiológicas y clínicas del TEC en niños de un hospital de Lima, Perú.

\section{EL ESTUDIO}

Se realizó una serie de casos retrospectiva. Se incluyeron pacientes menores de 14 años de edad, con diagnóstico de TEC al ingreso, hospitalizados en las salas de Pediatría, Unidad de Cuidados Intensivos Pediátricos o Emergencia Pediátrica del Hospital Nacional Cayetano Heredia, entre los años 2004-2011.

Se revisaron las historias clínicas y se recolectaron los datos en una ficha previamente diseñada. Se tomaron datos sociodemográficos además de las características del trauma, incluyendo hora, fecha, lugar y mecanismo. Se obtuvo la puntuación de la escala de Glasgow, tiempo de pérdida de conciencia, síndromes neurológicos asociados y estado de conciencia al ingreso. También se registraron la presencia de lesiones intracraneales y fracturas craneales. Los datos de la hospitalización que se recolectaron fueron: manejo en cuidados intensivos, tratamiento neuroquirúrgico, complicaciones médicas y tiempo de hospitalización. Para las condiciones de alta se consideró la categorización de sano, mejorado con signos neurológicos, y fallecido.

Elestudiofueaprobado porlos ComitédeÉticadel Hospital Nacional Cayetano Heredia y la Universidad Peruana Cayetano Heredia. El análisis se realizó calculando las frecuencias y proporciones de las variables cualitativas. De manera exploratoria se buscaron asociaciones entre algunas variables seleccionadas; para esto se utilizó la prueba de chi cuadrado en variables cualitativas y la prueba $U$ de Mann-Whitney en variables cuantitativas. Se usó el programa STATA versión 11 para el análisis estadístico.

\section{HALLAZGOS}

Durante el periodo de estudio se registraron 352 casos de TEC, de los cuales solo 316 contaban con datos

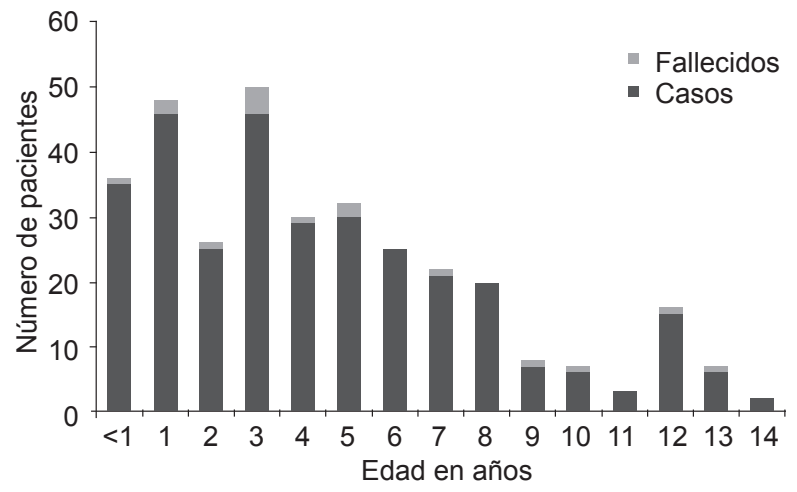

Figura 1. Casos y muertes por traumatismo encefalocraneano por años de edad, 2004-2011

completos disponibles, y 16 pacientes fueron registrados como fallecidos (Figura 1).

Se encontró una mediana de 37 casos por año (rango: 12 a 69). En los últimos cuatro meses del año ocurrieron el $39,0 \%(123 / 316)$ de los casos. La frecuencia de casos fue similar en todos los días de la semana, el $42,4 \%$ (134/316) ocurrió durante horas de la tarde.

La mediana de edad fue de $4 \pm 3$ años; según el sexo, 63,9\% (202/316) de los casos fueron hombres; $89,6 \%$ (283/316) vivían en Lima, y el resto fue referido de provincias. Las caídas correspondieron al $80,1 \%$ (253/316) de los accidentes, de estos, 164 fueron por caída libre a una altura promedio de 2,5 metros, 39 caídas fueron por rodamiento en escaleras y 12 al uso de bicicletas, patinetas o andadores. El lugar del accidente más frecuente fue el hogar, $59,2 \%$ de casos, siendo $94,7 \%$ (117/187) por caídas. En el examen clínico al ingreso $57,9 \%$ (183/316) de los casos presentó alteración de la conciencia donde fue más frecuente la somnolencia en $29,1 \%$ (92/316); en $22,5 \%$ (71/316) casos presentaron hipertensión endocraneana; las crisis convulsivas se registraron en $12,7 \%(40 / 316)$ casos de los cuales 10 presentaron convulsiones focales, 26 convulsiones generalizadas y 4 ambas; solo en $76,0 \%$ (240/316) historias se registró la escala de Glasgow al ingreso de los cuales 5,1\% (16/316) tuvo un puntaje menor a 9 . Según el diagnóstico por imágenes en $68,7 \%$ (217/316) se encontró una o más fracturas craneales siendo más frecuentes las fracturas lineales en $56,0 \%$ (177/316); las fracturas deprimidas y de base de cráneo se produjeron en $9,8 \%(31 / 316)$ y $5,7 \%(18 / 316)$ casos, respectivamente. La lesión intracraneal más común fue el hematoma epidural con $26,9 \%$ (85/316) (Tabla 1).

La mediana del tiempo de hospitalización fue $4 \pm 2$ días. Se requirió manejo en cuidados intensivos en $15,8 \%$ (50/316) de casos, e intervenciones neuroquirúrgicas 
Tabla 1. Características de los menores de 14 años con traumatismo encéfalocraneano atendidos en el Hospital Nacional Cayetano Heredia en Lima, Perú 2004-2011

\begin{tabular}{lcc}
\hline \multicolumn{1}{c}{ Características } & N & $(\%)$ \\
\hline Edad & $4^{*}$ & $(3)$ \\
\hline Sexo masculino & 202 & $(63,9)$ \\
\hline Procedencia & & \\
\hline Lima & 283 & $(89,6)$ \\
\hline Provincias & 33 & $(10,4)$ \\
\hline Tipo de accidente & & \\
\hline Caída & 253 & $(80,1)$ \\
\hline Tránsito vehicular & 33 & $(10,4)$ \\
\hline Golpe con objeto & 13 & $(4,1)$ \\
\hline Otro & 7 & $(2,2)$ \\
\hline No definido & 10 & $(27,8)$ \\
\hline Lugar del accidente & & \\
\hline Hogar & 187 & $(59,2)$ \\
\hline Calle & 53 & $(16,8)$ \\
\hline Colegio & 4 & $(1,3)$ \\
\hline Otro & 13 & $(4,1)$ \\
\hline No definido & 59 & $(18,7)$ \\
\hline Examen clínico al ingreso & & \\
\hline Alteración de conciencia & 183 & $(57,9)$ \\
\hline Hipertensión endocraneana & 71 & $(22,5)$ \\
\hline Crisis convulsiva & 40 & $(12,7)$ \\
\hline Glasgow menor a 9 & 16 & $(5,1)$ \\
\hline Estado de coma & 9 & $(2,9)$ \\
\hline Diagnósticos por imágenes & & \\
\hline Fracturas craneales & 217 & $(68,7)$ \\
\hline Hematoma epidural & 85 & $(26,9)$ \\
\hline Hematoma subdural & 28 & $(8,9)$ \\
\hline Hemorragia intraparenquimal & 24 & $(7,6)$ \\
\hline Hemorragia subaracnoidea & 15 & $(4,8)$ \\
\hline Hemorragia intraventricular & 4 & $(1,3)$ \\
\hline & & \\
\hline & & \\
\hline & & \\
\hline & & \\
\hline
\end{tabular}

*Media (desviación estándar)

en $14,2 \%(49 / 316)$ de casos. Al momento del alta $72,8 \%(230 / 316)$ de casos estaban completamente recuperados; $21,5 \%(68 / 316)$ estaba mejorado pero continuaba con algún signo neurológico, y en dos pacientes no se registró la condición de alta.

De los pacientes que sufrieron caídas, 80,6\% (204/253) se recuperó completamente; $17,4 \%$ (44/253) continuó con signos neurológicos, y 2,0\% (5/253) falleció. En pacientes que sufrieron accidentes de tránsito $39,4 \%$ (13/33) se recuperó completamente; 57,6\% (19/33) continuó con signos neurológicos y 1 falleció.

La presencia de fracturas lineales se asoció a la ocurrencia de hematomas epidurales $(p<0,01)$; las caídas se asociaron a una menor edad $(p<0,01)$ y la ocurrencia del accidente en casa $(p<0,01)$; los accidentes de tránsito se asociaron a una mayor edad $(p=0,03)$.

\section{DISCUSIÓN}

Se ha descrito al TEC en niños como una epidemia silente ${ }^{(14)}$ que requiere ser atendida eficientemente en todos los niveles de salud. En nuestro país no contamos con suficiente información sobre las características epidemiológicas y clínicas del TEC. Esta serie de casos se basa en pacientes procedentes de distritos aledaños al Hospital Nacional Cayetano Heredia y una minoría referida de provincias, lo que no permite determinar la magnitud del problema a nivel nacional, pero permite tener una idea de su espectro de gravedad.

Al igual que otras series latinoamericanas $(4,15)$ encontramos que el grupo etario más vulnerable fue el de niños menores de cinco años y que el principal mecanismo del trauma fueron las caídas en el hogar. En niños mayores los accidentes de tránsito fueron un mecanismo importante y frecuente. Estos hallazgos, sugieren que las campañas de prevención de accidentes en el hogar y de educación vial deberían ser una estrategia eficaz para disminuir la morbilidad del TEC.

EI TEC puede originar una gama de lesiones en la etapa aguda, las que producen diferentes patrones de daño en el encéfalo, tanto vascular, neuronal y glial ${ }^{(16)}$. El mayor volumen relativo de la cabeza y el centro de gravedad más alto, al igual que los factores del accidente como la altura de la caída y el tipo del suelo, son muy importantes para la extensión de la lesión (10). Además, en los recientes años se ha conocido mejor los mecanismos de daño secundario que se activan en respuesta al daño primario y explican la lesión por compromiso axonal, visible solo por resonancia magnética.

La asociación encontrada entre fracturas lineales y el hematoma epidural ponen en evidencia un mecanismo de gran impacto sobre el cráneo, que al provocar la fractura lacera la arteria meníngea media o sus colaterales provocando el subsecuente sangrado. La frecuencia encontrada fue similar a la reportada por Zopfi et al. (14) Sin embargo, en esta serie la cantidad de casos que requirieron manejo quirúrgico fue mucho menor, enfatizando que la mayoría de pacientes podrían evolucionar favorablemente con manejo médico únicamente.

En esta serie se presenta una gran variedad de lesiones y síndromes neurológicos, cuya gravedad se refleja en la necesidad de cuidados intensivos, básicamente para el manejo de la hipertensión endocraneana, crisis convulsivas y del compromiso severo del estado de conciencia. Los recursos técnicos disponibles en la actualidad permiten un monitoreo más cuidadoso de las diferentes complicaciones propias del TEC y de su comorbilidad ${ }^{(14,17,19)}$. 
La letalidad se encontró en el rango de las referencias internacionales, sin embargo, la mortalidad debería ser mayor, ya que se espera que $85 \%$ de fallecimientos ocurran antes de llegar al hospital ${ }^{(15)}$. Debido a la mayor frecuencia de TEC en niños de 1 a 5 años, proporcionalmente hubo más de la mitad de los fallecimientos en este grupo etario. En general, la mayoría de muertes ocurrieron en los primeros días de hospitalización, probablemente por una grave lesión secundaria y complicaciones infecciosas ${ }^{(20)}$.

Las lesiones craneoencefálicas pueden alterar las funciones motoras, sensoriales y cognitivas (21). Estos trastornos habitualmente toman meses y años para definirsecomosecuelas, mientrastanto, los niños requieren consultas, exámenes y terapias especializadas (22,23). A menudo durante este tiempo uno de los padres deja de trabajar o requieren de una tercera persona para el cuidado del niño. Todo esto ocasiona una carga, tanto emocional como económica, sobre la familia ${ }^{(23,24)}$. Por lo que el TEC pediátrico debería ser considerado como un problema de salud pública.

Dentro de las limitaciones en este estudio se encuentra la falta de un registro completo en las historias clínicas que fueron revisadas, existiendo un gran subregistro en la puntuación de Glasgow, lo que dificulta la valoración del paciente al ingreso. A pesar de esto, la distribución del puntaje de Glasgow en esta serie se asemeja a la de otros países ${ }^{(1,5,15,25)}$ y a una serie nacional realizada en un instituto especializado de niños ${ }^{(14)}$. Asimismo, por tratarse de un estudio retrospectivo en pacientes hospitalizados, la probabilidad de sesgos es alta y estos resultados no pueden ser extrapolados a la población general.

Si bien la epidemiología del TEC está muy estudiada en otros países, este estudio representa una primera experiencia nacional en un servicio de pediatría de un hospital nacional durante un periodo de ocho años, estos resultados son un primer paso para la implementación de medidas de prevención dirigidas a disminuir el impacto del TEC en la salud de la población pediátrica. Es necesaria la realización de más estudios que permitan determinar de manera objetiva la epidemiología y características clínicas del TEC en el Perú.

En conclusión, las caídas en el hogar fueron la principal causa de TEC donde los menores de 5 años son los más afectados y la lesión intracraneal más común fue el hematoma epidural.

Contribuciones de autoría: DGP ha participado en la concepción del estudio. AZV, DGM, MSK, CRO, LMV, EBP contribuyeron en la toma de los datos y AZV, DGM y MSK en el procesamiento de los datos. Todos los autores participaron en la redacción y revisión del artículo; todos aprobaron la versión final a publicar.

Fuente de financiamiento: autofinanciado.

Conflictos de interés: los autores declaran no tener conflictos de interés.

\section{REFERENCIAS BIBLIOGRÁFICAS}

1. Bruns J Jr, Hauser WA. The epidemiology of traumatic brain injury: a review. Epilepsia. 2003;44 Suppl 10:2-10.

2. Corrigan JD, Selassie AW, Orman JA. The epidemiology of traumatic brain injury. J Head Trauma Rehabil. 2010;25(2):72-80. doi: 10.1097/ HTR.0b013e3181ccc8b4.

3. Cooper KD, Tabaddor K, Allen-Hauser W, Shulman K, Feiner C, Factor P. The epidemiology of head injury in the bronx. Neuroepidemiology. 1983;2(12):79-88.

4. Boza C, Donoso A, Gigoux J, Camus A, Bruhn A, Valverde C, et al. Epidemiologia del traumatismo encefalocraneano en ninos del area suroriente de Santiago. Rev Chil Pediatr. 1997;68(2):83-7.
5. Annegers JF, Grabow JD, Kurland LT, Laws ER Jr. The incidence, causes, and secular trends of head trauma in Olmsted County, Minnesota, 19351974. Neurology. 1980;30(9):912-9.

6. Agramonte JS. Aspectos epidemiologicos en pacientes que sufrieron trauma pediatrico [Tesis para optar por el titulo de Cirujano Pediatra], Lima, Perú: Facultad de Medicina, Universidad Nacional Mayor de San Marcos; 2002.

7. Puvanachandra P, Hyder AA. Traumatic brain injury in Latin America and the Caribbean: a call for research. Salud Publica Mex. 2008;50(1):S3-5.

8. Eisele JA, Kegler SR, Trent RB, Coronado VG. Nonfatal traumatic brain injury-related hospitalization in very young children-15 states,
1999. J Head Trauma Rehabil. 2006;21(6):537-43.

9. Love PF, Tepas JJ 3rd, Wludyka PS, Masnita-Iusan C. Fall-related pediatric brain injuries: the role of race, age, and sex. J Trauma. 2009;67(Suppl 1):S12-5. doi: 10.1097/TA.0b013e3181ac7f22.

10. Gelfman MG, Ledesma J, Hauier F, Volonte P, Orbe G, Fiorentino J. Trauma por caída de altura en pediatría. Arch Argent Pediatr. 2005;103(5):414-9.

11. Tiret L, Hausherr E, Thicoipe M, Garros B, Maurette P, Castel JP, et al. The epidemiology of head trauma in Aquitaine (France), 1986: a community-based study of hospital admissions and deaths. Int J Epidemiol. 1990;19(1):133-40.

12. Tate RL, McDonald S, Lulham JM. Incidence of hospital-treated 
traumatic brain injury in an Australian community. Aust N Z J Public Health. 1998;22(4):419-23.

13. Rahimi-Movaghar V, Saadat S, Rasouli MR, Ghahramani M, Eghbali A. The incidence of traumatic brain injury in Tehran, Iran: a population based study. Am Surg. 2011;77(6):e112-4.

14. Zopfi RR, Ramirez E A, Toledo A M. Traumatismo encefalocraneano en niños: epidemiología. Rev Peru Neurocir. 2009;4(1):11-6.

15. Arango D, Quevedo A, Montes A, Cornejo W. Epidemiología del trauma encefalocraneano (TEC) en 90 lactantes mayores y preescolares atendidos en un servicio de urgencias pediátricas de tercer nivel, en Medellín, Colombia. Iatreia. 2008;21(3):271-9.

16. Atabaki SM. Pediatric head injury. Pediatr Rev. 2007;28(6):215-24.

17. Lowenstein D. Epilepsy after head injury: an overview. Epilepsia. 2009;50 Suppl 2:4-9. doi: 10.1111/j.15281167.2008.02004.x.
18. Liesemer K, Bratton SL, Zebrack CM, Brockmeyer D, Statler KD. Early posttraumatic seizures in moderate to severe pediatric traumatic brain injury: rates, risk factors, and clinical features. J Neurotrauma. 2011;28(5):755-62. doi: $10.1089 /$ neu.2010.1518.

19. Myhre MC, Grøgaard JB, Dyb GA, Sandvik L, Nordhov M. Traumatic head injury in infants and toddlers. Acta Paediatr. 2007;96(8):1159-63.

20. Keenan HT, Bratton SL. Epidemiology and outcomes of pediatric traumatic brain injury. Dev Neurosci. 2006;28(45):256-63.

21. Sancho-Rieger J,Parra-Martínez J. Actitud preventiva y terapéutica en las crisis epilépticas postraumáticas. Rev Neurol. 2002;35(Supl 1):S39-S42

22. Sesma HW, Slomine BS, Ding R, McCarthy ML. Executive functioning in the first year after pediatric traumatic brain injury. Pediatrics. 2008;121(6):e1686-95.

23. Aitken ME, McCarthy ML, Slomine BS, Ding R, Durbin DR, Jaffe KM, et al. Family burden after traumatic brain injury in children. Pediatrics. 2009;123(1):199-206. doi: 10.1542/ peds.2008-0607.

24. Rockhill CM, Fann JR, Fan MY, Hollingworth W, Katon WJ. Healthcare costs associated with mild traumatic brain injury and psychological distress in children and adolescents. Brain Inj. 2010;24(9):1051-60. doi: $10.3109 / 02699052.2010 .494586$.

25. Boza C, Donoso A,Gigoux J, Camus A, Bruhn A, Valverde C et al. Traumatismo encefalocraneano en ninos del area suroriente de Santiago. Rev Chil Pediatr. 1997;68(2):88-92.

Correspondencia: Daniel Guillén Pinto Dirección: Av. Honorio Delgado 430 San Martin de Porres

Teléfono: (511) - 3190000

Correo electrónico:dguillenpinto@gmail.com

\section{Consulte las ediciones anteriores de la Revista Peruana de Medicina Experimental y Salud Pública en wWw.scielosp.org}

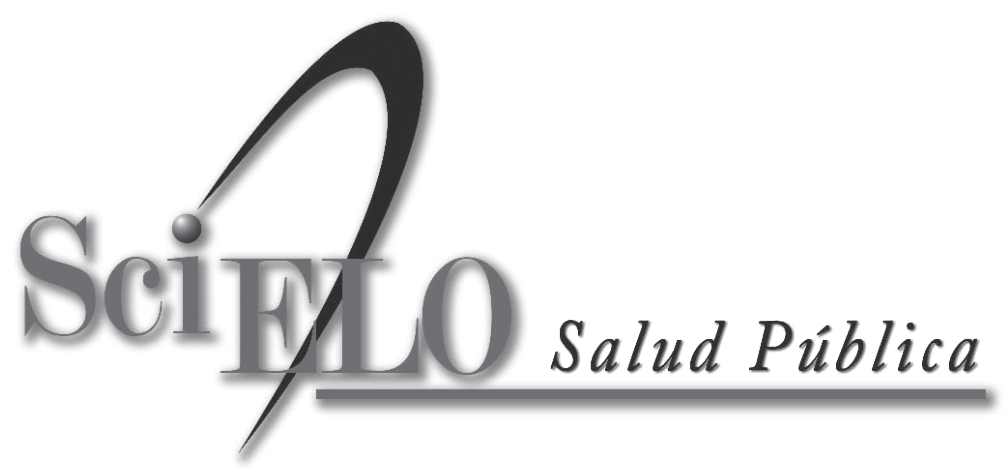

